ORIGINAL ARTICLE

\title{
Cinical and pathological characteristics of Chinese patients with antineutrophil cytoplasmic autoantibody associated systemic vasculitides: a study of 426 patients from a single centre
}

\author{
M Chen, F Yu, Y Zhang, M H Zhao
}

Postgrad Med J 2005;81:723-727. doi: 10.1136/pgmj.2005.034215

See end of article for authors' affiliations

Correspondence to: $\operatorname{Dr}$ M H Zhao, Renal Division and Institute of Nephrology, Peking University First Hospital Beiiing 100034, PR China; mhzhao@bjmu.edu.cn

Submitted

26 February 2005

Accepted 27 April 2005

\begin{abstract}
Background: Antineutrophil cytoplasmic autoantibodies (ANCA) are serological markers of ANCA associated systemic vasculitides (AASV), which is one of the most common multisystem autoimmune diseases. Features of Chinese patients with AASV have not been fully investigated.

Objective: To analyse the clinical and pathological characteristics of Chinese patients with AASV.

Methods: 426 Chinese patients with AASV diagnosed in the past eight years were retrospectively studied and their clinical and pathological data were analysed.

Results: Of the 426 patients, 87 (20.4\%) were Wegener's granulomatosis, 337 (79.1\%) were microscopic polyangiitis and two $(0.5 \%)$ were Churg-Strauss syndrome. Only 201 of $426(47.2 \%)$ patients were diagnosed within three months. Clinically, the patients had multisystem involvement. Altogether 371 of $426(87.1 \%)$ had kidney involvement and 260 of $426(61.0 \%)$ had lung involvement. The prevalences of renal involvement and fatigue were significantly higher in patients with MPO-ANCA than that in patients with PR3-ANCA; the prevalences of ophthalmic, nasal involvement, rash, and arthragia were significantly higher in patients with PR3-ANCA than those in patients with MPO-ANCA. The one and five year death rates were $13.1 \%$ and $22.4 \%$, respectively. The percentage of patients progressing to end stage renal disease at one and five years was $15.9 \%$ and $27.1 \%$, respectively.

Conclusions: AASV is not a rare autoimmune disease in Chinese people. Kidney and lung were the most vulnerable organs. For patients with multiorgan damage, an ANCA test should be performed to make an early diagnosis and to start treatment in time.
\end{abstract}

$\mathrm{V}$ asculitis is an inflammatory process of blood vessels, histopathologically characterised by vessel wall destruction and occlusion of the vascular lumen. An attempt to classify the diverse forms of vasculitis resulted in the Chapel Hill international consensus definition, which used the vessel size as the determinant of classification. ${ }^{1}$ American College of Rheumatology (ACR) has proposed classification criteria for some of the diseases. ${ }^{2}$ Wegener's granulomatosis (WG), microscopic polyangiitis (MPA), Churg-Strauss syndrome (CSS), and idiopathic rapidly progressive glomerulonephritis (iRPGN) are described as small vessel vasculitides and are acknowledged to be commonly associated with antineutrophil cytoplasmic autoantibodies (ANCA). They are termed as ANCA associated systemic vasculitides (AASV). Two fluorescence patterns of ANCAs are distinguished by indirect immunofluorescence (IIF), the cytoplasmic staining pattern (CANCA), and the perinuclear staining pattern (pANCA). Most patients with a cANCA pattern obtained by IIF have ANCAs directed against proteinase-3 (PR3), as determined by antigen specific ELISA. Patients with a pANCA mostly have ANCAs directed against one of a variety of antigens, such as myeloperoxidase (MPO). The combination of a cANCA with PR3-ANCA and pANCA with MPO-ANCA are specific for WG, MPA, or iRPGN. ${ }^{3}$

AASV must be recognised in time if successful treatment is to be implemented. Early diagnosis and appropriate immunosuppressive treatment are crucial for reducing the ability of acute vasculitis to cause death from major organ failure, for example, respiratory failure from pulmonary haemorrhage, and to reduce long term morbidity, for example, end stage renal failure.
AASV is one of the most common multisystem autoimmune diseases in the white population. A population based study from Norfolk, England, reported incidences of 8.5 cases per million for WG, 3.6 cases per million for MPA, and 2.4 cases per million for the CSS. ${ }^{4}$ In two large US cohorts studies of patients with $\mathrm{WG}^{5}{ }^{56}$ white people comprised more than $90 \%$ of all cases. Regional, geographical, ethnic, and seasonal differences in disease patterns and increasing incidence have been suggested, but lack confirmation. ${ }^{7}$ Studies from Norway suggested a doubling incidence of WG from 1992-1994 to 1995-1998. These figures probably reflect a real increase in incidence as well as improved awareness and diagnosis. AASV can occur at any age, especially elderly people.

The incidence of AASV in the Chinese population is unknown. In the early 1990s, after IIF-ANCA and ELISA using crude neutrophil acid extracts as solid phase ligands were established, a retrospective study of 50 hospitalised Chinese patients with crescentic glomerulonephritis or end stage renal disease showed that five of the 50 patients were ANCA positive. ${ }^{8}$ With the commercial IIF-ANCA kits being available and purified MPO and PR3 being used in routine ANCA screening assays, the prevalence of AASV increased substantially. In the past eight years, 426 patients with AASV were diagnosed in Peking University First Hospital. The

Abbreviations: ANCA, antinuetrophil cytoplasmic autoantibodies; AASV, ANCA associated systemic vasculitides; WG, Wegener's granulomatosis; MPA, microscopic polyangiitis; CSS, Churg-Strauss syndrome; ACR, American College of Rheumatology; ANA, antinuclear antibodies; ESR, erythrocyte sedimentation rate; MMF, mycophenolate mofetil; MPO, myeloperoxidase 
prevalence of ANCA and the antigen specificities, as well as demographic features of some of the Chinese patients with AASV was investigated. ${ }^{9}$ It was suggested that AASV might not be rare in the Chinese population. In this study, the clinical and pathological manifestations of the Chinese patients with AASV were investigated.

\section{METHODS \\ Patients}

A total of 426 patients with AASV, diagnosed from 1997 to 2004 in the Institute of Nephrology, Peking University First Hospital, were enrolled into this retrospective study. All the patients met the criteria of the Chapel Hill consensus conference definition (for WG, MPA, or CSS) and ACR classification criteria (for WG or CSS). Systemic lupus erythematosus, Henoch-Schölein purpura, rheumatoid arthritis were excluded. Acute renal failure was defined by the presence of progressively raised serum creatinine or $15 \%$ declined clearance rate of serum creatinine on the baseline within days or weeks. Clinical data were collected and analysed according to the organ involved.

\section{IIF assay to detect ANCA}

Standard IIF assay was performed accoding to the manufacturer (EUROIMMUN, Lübeck, Germany). Ethanol fixed human polymorphonuclear leucocytes (PMN) were used to detect ANCA and monkey liver sections were used to exclude antinuclear antibodies (ANA). cANCA and pANCA were distinguished according to staining patterns by two experienced technicians.

\section{Antigen specific ELISAs}

Two highly purified known ANCA antigens, PR3 and MPO, were used as solid phase ligands in ELISA, as previously reported. ${ }^{10}$

\section{Pathological examination}

Renal pathological data of 122 cases were evaluated using direct immunofluorescence, light and electron microscopy. Specimens of kidney were required to have a minimum of 10 glomeruli for classification of glomerulonephritis with light microscopy by the same renal pathologist.

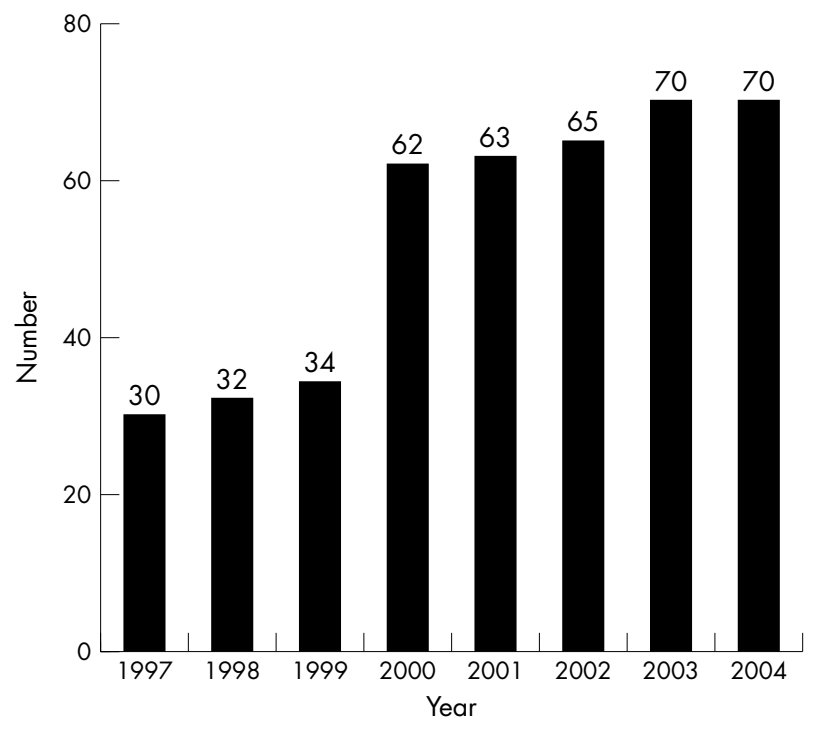

Figure 1 Patients with AASV diagnosed chronologically.

\section{Treatment protocols}

Induction treatment included corticosteroids and cyclophosphamide (CTX). Oral prednisone was prescribed at an initial dose of $1 \mathrm{mg} / \mathrm{kg} /$ day for four to six weeks, with reducing doses over time to 12.5 to $15 \mathrm{mg}$ by three months. CTX started on day 10-14 days after corticosteroids, daily oral $2 \mathrm{mg} / \mathrm{kg} /$ day or intravenous $0.7 \mathrm{~g} / \mathrm{m}^{2}$ every month. Patients with acute renal failure or pulmonary haemorrhage received three pulses of intravenous methylprednisolone $(7-15 \mathrm{mg} / \mathrm{kg} /$ day) before standard induction treatment. Some patients with severe pulmonary haemorrhage received additionally plasma exchanges. Azathioprine or mycophenolate mofetil (MMF) was given in maintenance treatment.

Remission was defined as the absence of clinical signs or symptoms or laboratory evidence of vasculitis activity. Relapse was defined as the return of clinical signs or symptoms or laboratory evidence of disease activity sufficient to warrant a sustained increase in immunosuppressive treatment.

\section{RESULTS}

\section{Prevalence and demographic features}

Of the 426 patients with AASV diagnosed from 1997 to 2004, 199 (46.7\%) were male and 227 (53.3\%) were female. Most of the patients were from northern China. There was no significant occupation of the patients. The average age was 56.1 (range 5-83) years old. Altogether 176 (41.3\%) cases were older than 65 years old and $38(8.92 \%)$ cases were younger than 20 years old. The number of diagnosed case increased chronologically (fig 1). Eighty seven patients $(20.4 \%)$ were WG, 337 (79.1\%) were MPA, and two $(0.5 \%)$ were CSS. Seventy (16.4\%) of these patients were positive for cANCA. Sixty nine of them could recognise PR3 only and the rest one could recognise both PR3 and MPO in antigen specific ELISA. Altogether 354 (83.1\%) patients were positive for pANCA with antigen specificity for MPO only in 346 (346 of 354,97.7\%) patients, and positive for both MPO and PR3 in eight cases ( 8 of $354,2.3 \%$ ). Two of 426 patients were positive for both pANCA and cANCA. One of them could recognise MPO only and the other could recognise both MPO and PR3 (table 1).

\section{Interval between onset of the disease and diagnosis}

The mean and median time between onset of the disease and testing for ANCA were 237.6 (ranging from 3 to 1460) and 60 days, respectively. Only 99 cases $(23.2 \%)$ were diagnosed within 30 days, $47.2 \%$ (201) cases within 90 days, 252 cases (59.2\%) within 180 days, $47(11.0 \%)$ cases more than one year, and one case was even diagnosed four years after the onset of the disease. There was no significant difference of the interval between onset of the disease and diagnosis between patients with PR3-ANCA and MPO-ANCA.

\section{Non-specific symptoms}

Of the 426 cases, $260(61.0 \%)$ had fatigue, $242(56.8 \%)$ had fever, 171 cases $(40.1 \%)$ had significant weight loss, 84 cases $(30.8 \%)$ had arthralgia, $110(25.8 \%)$ cases had muscle pain, and $78(18.3 \%)$ cases had a rash.

\section{Renal manifestations}

A total of 371 cases (371 of 426, $87.1 \%$ ) had renal involvement, of which $334(90.0 \%)$ had haematuria, 314 $(84.6 \%)$ had proteinuria, $307(82.7 \%)$ had raised serum creatinine with $229(61.7 \%)$ diagnosed to have acute renal failure, and only 31 cases $(8.36 \%)$ had nephrotic syndrome. Altogether 122 cases (32.9\%) had a renal biopsy, of which 38 $(31.1 \%)$ had focal segmental fibrinoid necrosis in glomeruli, $12(9.84 \%)$ had fibrinoid necrosis in arterioles, $83(68.0 \%)$ reached the criteria of crescentic glomerulonephritis (over 
Table 1 ANCA results of serum samples in IIF and ELISA

\begin{tabular}{lllll}
\hline & anti-PR3 & anti-MPO & anti-PR3 and MPO & Total \\
\hline cANCA & 69 & 0 & 1 & 70 \\
pANCA & 0 & 346 & 8 & 354 \\
cANCA and pANCA & 0 & 1 & 1 & 2 \\
Total & 69 & 347 & 10 & 426 \\
\hline
\end{tabular}

$50 \%$ glomeruli had large crescents formation), and 11 $(9.02 \%)$ had granulomatous lesion in renal interstitium.

\section{Pulmonary manifestations}

Two hundred and sixty cases (260 of $426,61.0 \%$ ) had lung symptoms such as cough, often with sputum production, of which $104(40.0 \%)$ had haemoptysis. One hundred and fifty cases had alveolar infiltrates or interstitial changes on chest films or computed tomography, of which 130 cases $(86.7 \%)$ had shadows in one or both lungs and 79 (52.7\%) had pulmonary interstitial fibrosis, multiple nodules, or cavitations.

\section{Manifestations of other organs}

Of the 426 patients, 83 cases (19.5\%) had ophthalmic disease manifested as conjunctivitis, keratohelcosis, episcleritis and uveiitis, optical nerve vasculitis, and declining vision. Ninety nine cases $(23.2 \%)$ had ear involvements, manifested as tinnitus, perforation of the tympanic membrane, and declining or loss of hearing. Thirty five cases $(8.22 \%)$ had nasal involvement, manifested as sinusitis, stuffiness, epistaxis, and brown or bloody crusts, rhinitis, septal erosions, or even septal perforation. One hundred and eighty three cases $(43.0 \%)$ had gastrointestinal involvement manifested as nausea, vomiting, anorexia, abdominal pain, loose bowels, or bloody faeces. Five patients had a gastrointestinal ulcer. Sixty seven cases (15.7\%) had neurological disorders such as peripheral mononeritis multiplex, dizziness, headache, and coma. Three patients has cerebral haemorrhage. One patient had Guillain-Barre syndrome (fig 2).

Further analysis showed that the prevalences of renal involvement and fatigue were significantly higher in patients with MPO-ANCA than that in patients with PR3-ANCA $\left(90.2 \%\right.$ v 79.7\%, $\chi^{2}=6.21, \mathrm{p}<0.05 ; 63.7 \%$ v $46.4 \%, \chi^{2}=7.24$, $\mathrm{p}<0.01$, respectively); the prevalences of ophthalmic, nasal involvement, rash, and arthragia were significantly higher in patients with PR3-ANCA than those in patients with MPOANCA $\left(31.9 \% \vee 16.7 \%, \chi^{2}=8.53, \mathrm{p}<0.01 ; 21.7 \% \quad v 5.76 \%\right.$, $\chi^{2}=19.1, \mathrm{p}<0.01 ; 27.5 \%$ v $16.4 \%, \chi^{2}=4.76 \%, \mathrm{p}<0.05 ; 43.5 \%$ $v 28.0 \%, \chi^{2}=6.54, \mathrm{p}<0.05$, respectively) (table 2 ).

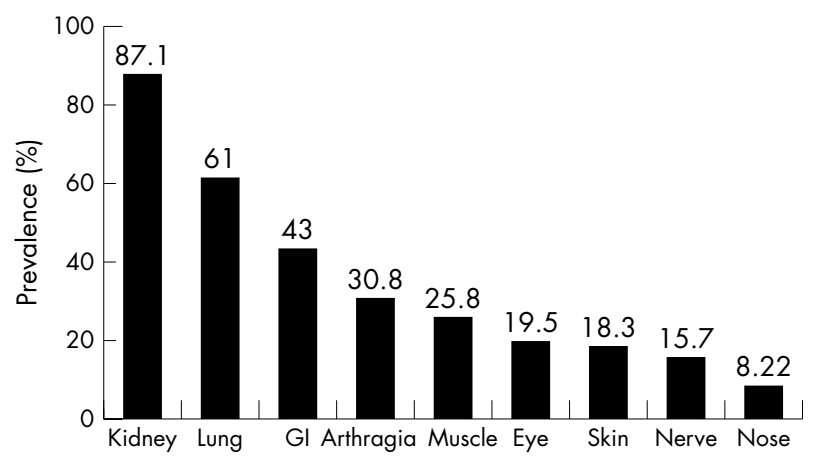

Figure 2 Prevalences of organ involvement.

\section{Laboratory findings}

A total of 300 of 337 cases $(89.0 \%)$ had anaemia, of which only 83 cases $(27.7 \%)$ had mild anaemia with haemoglobin ( Hb) over 90 g/l, 172 cases $(57.3 \%)$ had moderate anaemia with $\mathrm{Hb}$ between 60 and $90 \mathrm{~g} / \mathrm{l}$, and 45 (15.0\%) had severe anaemia with $\mathrm{Hb}$ less than $60 \mathrm{~g} / \mathrm{l}$. Altogether 125 of 287 $(43.6 \%)$ cases had leucocytosis and 56 of $195(28.7 \%)$ had thrombocytosis. A total of 265 of 285 cases $(93.0 \%)$ had raised erythrocyte sedimentation rate (ESR), of which 92 $(34.7 \%)$ were over $100 \mathrm{~mm} \mathrm{lst} \mathrm{h}$ and $178(67.2 \%)$ were over $60 \mathrm{~mm}$ lst h. Altogether 132 of 185 cases $(71.4 \%)$ had raised $\mathrm{C}$ reactive protein. No significant difference was found in these laboratory findings between PR3-ANCA and MPOANCA positive patients.

\section{Outcomes}

A total of 44 of $130(33.8 \%)$ and 86 of $130(66.2 \%)$ cases received prednisone together with daily oral or monthly intravenous CTX, respectively. After the induction phase treatment, 105 of $130(88.5 \%)$ cases had complete or partial remission. One hundred and seven cases were followed up. The average duration of follow up was 29.7 (1-108) months. A total of 24 of $107(22.4 \%)$ patients died and 29 of 107 $(27.1 \%)$ patients progressed to end stage renal disease and received renal replacement treatment. The one and five year death rates were $13.1 \%$ and $22.4 \%$, respectively. The percentage of patients progressing to end stage renal disease at one and five years was $15.9 \%$ and $27.1 \%$ respectively.

\section{DISCUSSION}

AASV is one of the most common multisystem autoimmune diseases in the white population. It is an important cause of mortality (if untreated) and morbidity (despite current aggressive treatment). The annual incidence and point prevalence of renal vasculitis in Europe is 10-20/million/year and 150-200/million, respectively. ${ }^{71}$ In China, after improved awareness and diagnostic techniques, more patients with AASV were diagnosed in recent years, but there is lack of systemic investigation on incidence. Over 400 patients with AASV were diagnosed in our centre in the past eight years and the number of patients increased chronologically. It was suggested that AASV was not rare in China.

In this study, it was found that the average age of Chinese patients with AASV was 56.1 (ranging from 5 to 83) years old. Over $40 \%$ of the cases were more than 65 years old, and nearly 1 of 10 of the cases were younger than 20 years old, showing that AASV could affect people of all age-elderly people were more susceptible, but children and teenagers were not rare. This is consistent with our previous studies and similar to the white population..$^{11-14}$

MPO and PR3 are the two important target antigens of ANCA for Chinese patients with AASV, which is consistent with the epidemiology reports in white populations. ${ }^{75}$ However, in our study, patients with MPA were about four times as many as patients with WG. Substantial evidences suggested that throughout Europe, there is a different proportion of WG and MPA. In northern Europe and Germany there are many more patients with WG than MPA, whereas in southern Europe, a study from Spain 
Table 2 Clinical manifestations of 426 patients with AASV

\begin{tabular}{|c|c|c|c|}
\hline & Number $(\%)$ & PR3-ANCA $(n=69)$ & MPO-ANCA $(n=347)$ \\
\hline Fever & $242(56.8)$ & $44(63.8)$ & $190(54.8)$ \\
\hline Fatigue ${ }^{* *}$ & $260(61.0)$ & $32(46.4)$ & $221(63.7)$ \\
\hline Weight loss & $171(40.1)$ & $23(33.3)$ & $144(41.5)$ \\
\hline Muscle pain & $110(25.8)$ & $18(26.1)$ & $92(26.5)$ \\
\hline Arthragia* & $131(30.8)$ & $30(43.5)$ & $97(28.0)$ \\
\hline Rash* & $78(18.3)$ & $19(27.5)$ & 57 (16.4) \\
\hline Renal* $^{*}$ & $371(87.1)$ & 55 (79.7) & $313(90.2)$ \\
\hline Pulmonary & $260(61.0)$ & $38(55.1)$ & $217(62.5)$ \\
\hline Ophthalmic** & 83 (19.5) & 22 (31.9) & $58(16.7)$ \\
\hline Ear & $99(23.2)$ & $21(30.4)$ & 74 (21.3) \\
\hline Nasal$^{* *}$ & $35(8.22)$ & $15(21.7)$ & $20(5.76)$ \\
\hline Gastrointestinal & $183(43.0)$ & $24(34.8)$ & $157(45.2)$ \\
\hline Nerve system & 67 (15.7) & $11(15.9)$ & $55(15.9)$ \\
\hline
\end{tabular}

suggested that patients with MPA were about two to three times as many patients with WG. ${ }^{11}{ }^{16-18}$ This may be attributed to the geographical, environmental, or genetic differences as there had been evidence that geographical and genetic factors both played a part in the pathogenesis of AASV. The preponderance of MPA might be one of the epidemiological characteristics of Chinese patients with AASV.

Kidney and lung are the two most vulnerable organs to be involved in patients with AASV. In this study, nearly $90 \%$ of cases had renal involvement and more than $60 \%$ of them had acute renal failure, which might be the major cause for long term end stage organ damage. However, the high prevalence of renal involvement might be to some extend a result of selective bias, because most patients with renal diseases were referred to us. Another possible reason might be the unawareness of the systemic disease by physicians and thus resulted in late diagnosis and increased prevalence of renal involvement. The prevalence of renal involvement and acute renal failure reported by the respiratory department in our hospital was $76.9 \%$ (10 of 13 ) and $38.5 \%$ (5 of 13), respectively. ${ }^{19}$ More than $60 \%$ had pulmonary involvement with $40 \%$ having pulmonary haemorrhage, which was the major cause of acute respiratory failure or death. Although $43 \%$ and $15.7 \%$ of the cases had gastrointestinal and nervous involvement respectively, they were often neglected by clinicians and these might be under-evaluated in this retrospective study. Besides visceral vasculitis, about $8 \%$ to $23 \%$ also suffered from auditory, ophthalmic, and nasal involvements. All the above mentioned findings showed that AASV is characterised by multisystem injury.

Although cANCA/PR3-ANCA was mainly found in patients with WG and pANCA/MPO-ANCA was mainly found in patients with MPA and iRPGN, it was not exclusive. Our study also found that there were some difference in the clinical manifestations between patients with PR3-ANCA and MPO-ANCA. The prevalences of fatigue and renal involvement were significantly higher in patients with MPO-ANCA than those in patients with PR3-ANCA. The prevalences of ophthalmic, nasal invlovement, rash, and arthragia were significantly higher in patients with PR3-ANCA than those in patients with MPO-ANCA. Some authors argued that one of the possible reasons for higher proportion of renal disease in patients with MPO-ANCA positive might be attributable to the delay of diagnosis. More patients with PR3-ANCA positive had upper respiratory and ophthalmic involvements, which probably reduce the patients' and doctors' delay. Earlier diagnosis and start of treatment might reduce the frequecy of renal involvements. ${ }^{20-23}$ In this study, however, there is no significant difference of the intervals between onset of disease and diagnosis between PR3-ANCA and
MPO-ANCA groups. Whether there are different mechanisms in the pathogenesis of renal disease in these ANCA subsets remains unclear.

Aberrant laboratory findings suggestive of active vasculitis included severe anaemia unparalleled with the degree of renal failure or pulmonary haemorrhage, or both, leucocytosis, thrombocytosis, increased non-specific inflammatory markers such as ESR and $\mathrm{C}$ reactive protein. ${ }^{24}$ The laboratory findings of our patients showed that most of them were in active phase at the time of diagnosis.

This study also preliminarily analysed the outcome of the patients. However, there were many patients lost during follow up, so the actual mortality and rate of end stage renal disease might be much higher.

The prognosis of untreated AASV is poor, with up to $90 \%$ of patients dying within two years. ${ }^{5}$ Early diagnosis is crucial, otherwise life threatening disease such as acute renal failure or respiratory failure may develop quickly. In this study, unfortunately, more than $40 \%$ of the patients were diagnosed six months after onset of the disease, and more than $10 \%$ of the cases were more than one year, which would undoubtedly worsen the prognosis. This showed that the awareness of this disease still needs to be improved in clinicians. Vassilopoulos $^{25}$ reported that in patients with severe multiorgan dysfunction or pulmonary disorders, there was a high prevalence of ANCA. Therefore, for the suspected patients, prompt ANCA detection is crucial for the early diagnosis of AASV.

\section{CONCLUSION}

AASV is not rare in the Chinese population. Renal and pulmonary involvement was common and life threatening. For patients with multisystem involvement, ANCA testing using both IIF and antigen specific ELISA is recommended and it provides a helpful tool facilitating earlier diagnosis.

\section{ACKNOWLEDGEMENTS}

We are grateful to Dr Yanping Peng for assistance in collecting the data of the patients.

\author{
Authors' affiliations \\ M Chen, F Yu, Y Zhang, M H Zhao, Renal Division and Institute of \\ Nephrology, Peking University First Hospital, Beijing, PR China \\ Funding: none. \\ Conflicts of interest: none.
}

Ethics approval and patient consent: this research was approved by the ethnics committee of Peking University First Hospital (Peking University First Hospital, Beijing 100034, PR China) and with informed consent of patients. 


\section{REFERENCES}

1 Jennette JC, Falk RJ, Andrassy K, et al. Nomenclature of systemic vasculitides: the proposal of an international consensus conference. Arthritis Rheum 1994;37:187-92.

2 Hunder GG, Arend WP, Bloch DA, et al. The American College of Rheumatology 1990 criteria for the classification of vasculitis. Arthritis Rheum 1990:33:1088-93.

3 Hagen EC, Daha MR, Hermans J, et al. Diagnostic value of standardized assays for anti-neutrophil cytoplasmic antibodies in idiopathic systemic vasculitis. EC/BCR project for ANCA assay standardization. Kidney Int 1998:53:743-53

4 Watts RA, Carruthers DM, Scott DG. Epidemiology of systemic vasculitis: changing incidence or definition? Semin Arthritis Rheum 1995;25:28-34.

5 Hoffman GS, Kerr GS, Leavitt RY, et al. Wegener granulomatosis: an analysis of 158 patients. Ann Intern Med 1992;116:488-98.

6 Stone JH. Wegener's Granulomatosis Etanercept Trial Research Group. Limited versus severe Wegener's granulomatosis: baseline data on patients in the Wegener's granulomatosis etanercept trial, Arthritis Rheum 2003;48:2299-309

7 Watts RA, Scott DG. Epidemiology of the vasculitides. Curr Opin Rheumatol 2003; 15:11-16.

8 Zhao MH, Wang HY, Zhang YK. The renal involvement of primary vasculitis: clinical and pathological analysis of 5 cases. Chin J Intern Med 1993;32:40-3.

9 Xin G, Zhao MH, Wang HY. Detection rate and antigenic specificities of antineutrophil cytoplasmic antibodies in Chinese patients with clinically suspected vasculitis. Clin Diagn Lab Immunol 2004;1 1:559-62.

10 Zhao MH, Lockwood CM. A comprehensive method to purifiy three majo ANCA antigens: proteinase 3, myeloperoxidase and bactericidal/ permeability- increasing protein from human neutrophil granule acid extract. J Immunol Meth 1996;197:121-30.

11 Gonzalez-Gay MA, Garcia-Porrua C, Guerrero J, et al. The epidemiology of the primary systemic vasculitides in northwest Spain: implications of the Chapel Hill consensus conference definitions. Arthritis Rheum 2003;15:388-93.

12 Booth AD, Almond MK, Burns A, et al. Outcome of ANCA-associated renal vasculitis: a 5-year retrospective study. Am J Kidney Dis 2003;41:776-84.
13 Wang Y, Zhao MH, Yu J, et al. The clinical and pathological characters of Chinese elderly patients with anti-neutrophil cytoplasmic autoantibodies associated small vasculitis. Exp Geronto 2004;39:1401-5.

14 Yu F, Zhao MH, Huang JP, et al. Clinical and pathological features of antineutrophil antibody associated systemic vasculitis in children. Zhonghua Er Ke Za Zhi 2003;41:831-4

15 Kamesh L, Harper L, Savage COS. ANCA-positive vasculitis. J Am Soc Nephrol 2002;13:1953-60.

16 Watts RA, Lane SE, Scott DG, et al. Epidemiology of vasculitis in Europe. Ann Rheum Dis 2001;60:1156-7.

17 Watts RA, Scott DGI. Epidemiology of the vasculitides. Curr Opin Rheumatol 2003;15:11-16.

18 Reinhold-Keller E, Herlyn K, Wagner-Bastmeyer R, et al. No difference in the incidences of vasculitides between north and south Germany: first results of the German vasculitis register. Rheumatology 2002;41:540-9.

$19 \mathrm{Li} \mathrm{H}, \mathrm{He} \mathrm{B}, \mathrm{Zhu} \mathrm{Y}$, et al. Diagnosis and treatment of primary small vessel vasculitis with involvement of lungs. Chin J Tuberc Respir Dis 1999;22:347-50.

20 Bajema IM, Hagen EC, Hermans J, et al. Kidney biopsy as a predictor for renal outcome in ANCA-associated necrotizing glomerulonephritis. Kidney Int 1999:56:1751-8.

21 Franssen C, Gans R, Kallenberg C, et al. Disease spectrum of patients with antineutrophil cytoplasmic autoantibodies of defined specificity: distinct differences between patients anti-proteinase 3 and anti-myeloperoxidase autoantibodies. J Intern Med 1998;244:209-16.

22 Franssen CF, Stegeman CA, Kallenberg CG, et al. Antiproteinase 3 and antimyeloperoxidase-associated vasculitis. Kidney Int 2000;57:2195-206

23 Hauer HA, Bajema IM, van Houwelingen, et al. Renal histology in ANCAassociated vasculitis: Differences between diagnostic and serologic subgroups. Kidney Int 2002;61:80-9.

24 Tse WY, Cockwell P, Savage COS. Assessment of disease activity in systemic vasculitis. Postgrad Med J 1998;74:1-6.

25 Vassilopoulos D, Niles JL, Villa Forte A, et al. Prevalence of antineutrophil cytoplasmic antibodies in patients with various pulmonary diseases or multiorgan dysfunction. Arthritis Rheum 2003;49:151-5.

\section{1 th European Forum on Quality Improvement in Health Care}

26-28 April 2006, Prague, Czech Republic

For further information please go to: www.quality.bmipg.com

Book early to benefit from a discounted delegate rate 